\title{
Synthesis and Processing of Nanostructured Films, and Introduction to and Comparison with Plasma Electrolysis
}

\section{1}

Why Nanostructures Are Important

Nanostructures have a volume that is intermediate between molecular and microscopic (dimensions in micrometers) structures. It is essential to make a distinction between the number of dimensions that are on the nanometric scale. A planar nanostructure has one dimension on the nanometric scale, the surface depth being between 1 and $100 \mathrm{~nm}$. A nanotube has two dimensions on the nanometric scale, the diameter being between 1 and $100 \mathrm{~nm}$, while the other dimension (the length) may be much larger. Finally, well-separated nanopowders have three dimensions on the nanometric scale, the dimension of a nanoparticle being between 1 and $100 \mathrm{~nm}$ in each arbitrary direction. The terms "nanoparticle" and "ultra-fine particle" are usually used with the same meaning, but the dimensions of ultra-fine particles are usually greater than those of nanoparticles [1].

As an interesting example of nanostructures, different nanostructures of carbon such as fullerenes, nanotubes, nanocones and graphene have exclusive mechanical and physical properties. Their superior properties are related to their firm skeletons created by bonded planar orbitals sandwiched between overlaid unsaturated bonds. Small atoms such as boron, nitrogen, and so on can diffuse among or replace the atoms of these nanostructures to increase their various properties or create locally active sites. Carbon nanostructures can also be chemically treated to achieve other activities, especially catalytic activities. Recently, some investigations have suggested that nitrogen-diffused carbon nanotubes will show enough electrocatalytic activity for reduction of oxygen. These treatments also become very attractive by forming stable metal-diffused carbon nanostructures for applications with catalytic activities [2].

Considering these attractive potential usages of nanostructures, interest in their application is growing increasingly. The fabrication methods of nanostructures allow us to arrange their atoms in nanometric size. One nanometer is about equal to the sum of the diameters of four atoms and also approximately 50000 times smaller than a human hair. Considering the time you spend to read these sentences, your 
fingernails will approximately grow about one nanometer. An attractive target of fabrication methods of nanostructures is their self-assembly on the nanometric scale and thus the production of large amounts of new materials with superior properties. Connecting nanostructures to microstructures and also to bigger structures by such desired self-assembly can be done to create large assemblies.

The applications of nanostructures are very wide, from nanometric devices in computer chips to the coatings of giant industrial components. Nanolayers can show superior magnetoresistance properties, which makes them suitable as a material for the heads of hard disks. Nanowires could be used as a critical component in the next generation of computers. They will cause the speed of these computers to be limited not by transistor switching time but by the travel time of the signals along these wires. Nanostructures also can increase the data storage capacity in related devices to more than a million times the recent usual capacities. Atoms can be located in completely well-defined directions and occupy just a space on the nanometric scale. Such data storage can be controlled on the nanometric scale by considering the presence of an atom as a " 1 " (or "on" position) and its absence as a "0" (or "off" position). Reaching a device that works with one atom per bit can be considered as the final target [3].

Another application of nanostructures can be seen in some of nanostructured zeolites. They have a channel with opening size around $0.5 \mathrm{~nm}$. Their small size of nanopore makes them suitable for the separation of very small molecules by taking into account the difference in their size. These meso- and microporous structured materials are widely used in the refinery industries for their catalytic, adsorbent and ion exchange properties. Their considerable catalytic activity and the selection of a specific zeolite are related to the large interior surface area and well-distributed active sites. On the other hand, nanowires and nanorods have been investigated for their exclusive properties, with potential uses in catalysis, sensors, and nano-electromechanical devices. New methods for the synthesis of different semiconductors have extended the application of nanowires to optics and actuators, making them a preferable building block for nanodevices [4].

What is so interesting about nanostructures that has attracted a lot of researches during recent years? It can be summarized as the term "size effect." Consider a piece of semiconductor and its bandgap, which is its important property for electronicrelated applications. Decreasing its dimensions to smaller pieces with $0.1 \mu \mathrm{m}$ $(100 \mathrm{~nm})$ average length will not influence the bandgap, but further decrease of its dimensions to smaller pieces with average dimensions less than $10 \mathrm{~nm}$ will change its bandgap and cause it to show some new properties such as visible light or enough catalytic activity for a specific reaction. These new properties are related to the "size effect," and $100 \mathrm{~nm}$ is approximately the border: nanostructures with dimensions lower than this amount will be affected by the size effect. The reasons for the different properties of materials on the nanometric scale or their nanometric size effect have been investigated through much research in recent years and have created many industrial applications of nanostructures [5].

Looking at it from the point of view of fabrication methods for nanostructures, one will notice that different methods have different capabilities to produce specific characteristics. For example, the distribution of nanocrystallites in a nanostructure 
or the distribution of hard nanoparticles in a nanocomposite can be controlled exactly by some methods whereas other ones cannot control it exactly. Some of them can operate at room temperature while other ones just work at high temperatures, and this will restrict their potential usage for industrial applications. This limitation will also affect the application of methods that work under high-vacuum conditions from an economic point of view [6, 7].

Plasma electrolysis [8-10], which has attracted a lot of researchers in recent years, has shown itself to be an interesting method for the fabrication of different nanostructures. This method has great ability to fabricate different kinds of nanostructures, with a few limitations that have been mentioned. This atmospheric-based method consists of applying a relatively high potential to an immersed component in a liquid at room temperature, and mainly its cathodic branch can produce nanostructures with desirable distribution and properties. Similar trends in change in properties of nanostructures fabricated by this method with their characteristics of nanocrystallites or nanoclusters have been proved through different researches. Figure 1.1 illustrates the polarization resistance of nanostructured carbide-based layers fabricated by cathodic plasma electrolysis. The uniform decrease of polarization resistance due to increasing the average size of nanocrystallites can be seen in this figure.

Different kinds of nanometric size effect can be seen in nanostructures that have been fabricated by plasma electrolysis. The changing amount and changing trend of these size effects can be controlled by precise adjustment of the effective factors of this method. For example, in the fabrication of nanostructured carbidebased layers by cathodic plasma electrolysis using monopolar pulsed current, increasing frequency and decreasing duty cycle will lead to decreasing average nanocrystallite size produced and increasing properties such as corrosion resistance and wear resistance.

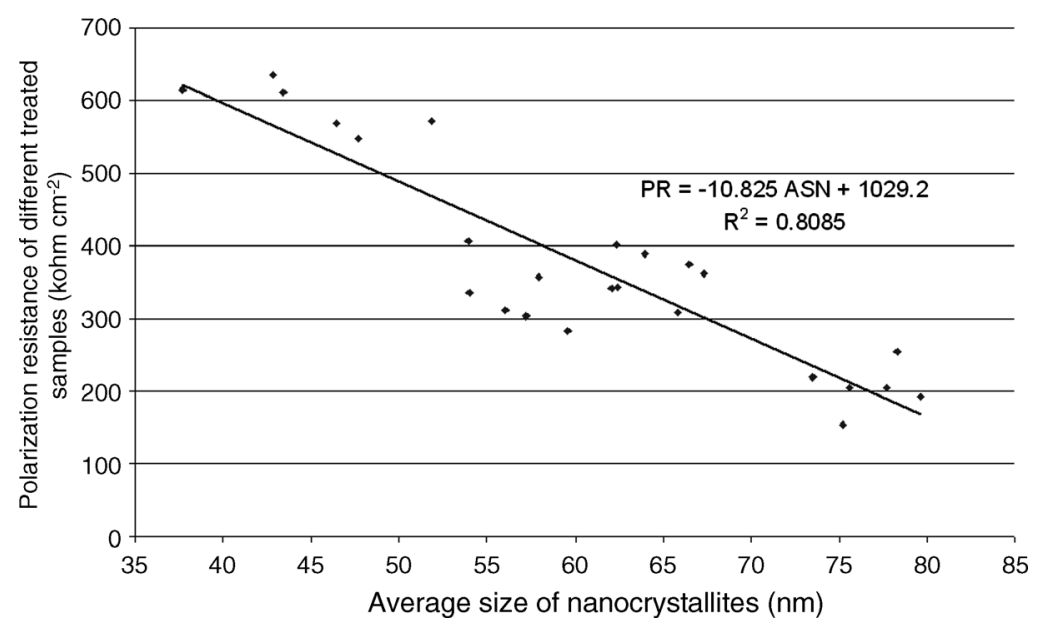

Figure 1.1 Polarization resistance of different treated samples by plasma electrolysis versus the average size of nanocrystallites [11]. 
1.2

\section{Different Types of Nanostructures}

There are different classifications of nanostructures in nanotechnology. Nanostructures usually classified by their geometrical properties. Nanostructures usually consist of nanocages, nanocrystallites, nanobelts, nanoneedles, nanocomposites, nanofabrics, nanofibers, nanoflakes, nanoflowers, nanofoams, nanomeshes, nanoparticles, nanopillars, nanopin films, nanorings, nanorods, nanoshells, nanopowders, nanoclusters, nanowires, nanotubes, quantum dots, quantum heterostructures and sculptured thin films $[12,13]$.

Classifying nanostructures according to their dimensions is the most popular mode of their classification. As shown in Figure 1.2, nanostructures can be described as zero- (0-D), one- (1-D), two- (2-D), and three-dimensional (3-D) nanomaterials.

0 -D

All dimensions $(x, y, z)$ at nanometric scale

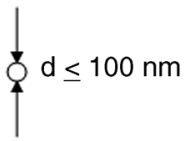

Example:

Nanoparticles

$1-\mathrm{D}$

Two dimensions $(x, y)$ at nanometric scale

One other dimension $(\mathrm{L})$ is not

Example:

Nanorods, Nanotubes

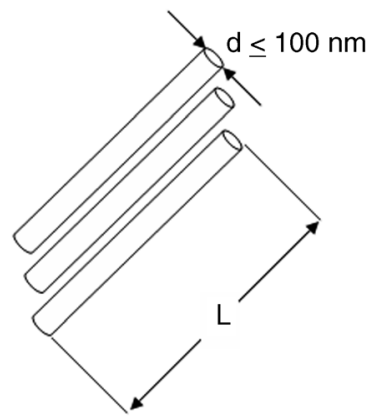

2-D

One dimension ( $\mathrm{t}$ ) at nanometric scale

Two other dimensions $\left(L_{x}, L_{y}\right)$ are not

Example:

Thin nanofilms

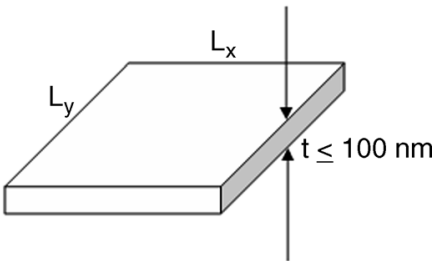

3-D

All of three dimensions $\left(L_{x}, L_{y}, L_{z}\right)$ are not at nanometric scale

Example:

Nanocrystalline and nanocomposite materials

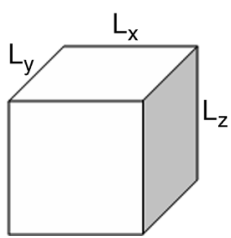

Figure 1.2 Classification of nanostructures according to 0-D, 1-D, 2-D, and 3-D. 
Some researchers also add amorphous materials as another branch for this classification. This classification considers the dimensions of nanostructure that are not located in the nanometric size range $(<100 \mathrm{~nm})$.

All of the dimensions of 0-D nanostructures are in the nanometric size range (such as nanoparticles or well-separated nanopowders). The 1-D nanostructures have a dimension that is outside the nanometric size range. These 1-D nanostructures have a shape like a rod, and consist of nanotubes, nanorods, nanoneedles, and nanowires. The 2-D nanostructures have two dimensions outside of the nanometric size range. Hence these 2-D nanostructures display plane-like structures, and consist of thin films, nanocoatings, and nanolayers. The 3-D nanostructures have three dimensions outside of the nanometric size range. These bulk 3-D nanostructures consist of many various kinds themselves, and usually comprise nanocrystalline units that show the affected properties of nanoscale due to the size effect. A 3-D nanostructure can include different distributions of nanoparticles or nanocrystallites, groups of nanowires and nanotubes, and also different nanolayers. All of the 0-D, 1-D, 2-D and 3-D nanostructures can be amorphous or nanocrystalline.

The 2-D and 3-D nanostructures can also be classified in more detail. The simplest shape of a 2-D nanostructure is a plane with a depth below $100 \mathrm{~nm}$ and other dimensions larger than nanometric dimensions. In spite of its external dimensions, this plane can show interior nanostructural dimensions, for example, nanocrystals (or nanograins) with nanoscale dimension. This 2-D nanocrystalline layer may exhibit some new size-related properties different from those of a microcrystalline layer. If the external thickness remains in the nanometric size range, the interior grains (crystallites) can be outside of the nanoscale and yet still categorized as a nanostructured material. So the interior structural dimensions (for grains or crystallites) and exterior surface dimensions (exclusively its depth) can be in the nanometric size range, and this difference will divide the 2-D nanostructures as internal nanostructured and non-internal nanostructured layers.

The dimensions of 3-D nanostructures will not be located in any nanometric size range but they still show such size-affected properties. For example, 3-D nanocomposites can be produced with two or more phases with different properties and their total synergistic properties cannot be reached by each phase alone. The matrix of the nanocomposite has dimensions bigger than nanometric scale and the reinforcing material is generally at the nanometric size range. These 3-D nanostructures can be classified by the type of reinforcing phases, for example, nanoparticles, nanorods, nanotubes or nanoflakes. Also the 3-D nanostructure can be nanocrystalline or microcrystalline in its internal structure, such as mentioned for 2-D nanostructures. Table 1.1 shows the general features and different classes of nanostructures with their dimensionality.

The position of nanostructures in engineering materials can also be seen in Figure 1.3. The overlapping zones in this figure are related to binary and tertiary composite materials. All of the engineering materials can be nanostructures. The areas of zones covered by the triangle of nanostructures cannot be compared with each other but the overall coverage of the different circles and the triangle show the position of nanostructures among engineering materials. 
Table 1.1 General features and different classes of nanostructures.

\begin{tabular}{llll}
\hline Dimensionality & \multicolumn{2}{c}{ General feature } \\
\cline { 2 - 4 } & $\begin{array}{l}\text { Separated } \\
\text { nanomaterials }\end{array}$ & $\begin{array}{l}\text { Surface } \\
\text { nanomaterials }\end{array}$ & $\begin{array}{l}\text { Bulk } \\
\text { nanomaterials }\end{array}$ \\
\hline 0-D & Well-dispersed & $\begin{array}{l}\text { Nanocrystalline } \\
\text { thin layers } \\
\text { 1-D }\end{array}$ & Nano connections \\
2-D & $\begin{array}{l}\text { Nanorods and } \\
\text { nanotubes } \\
\text { Thin nanofilms }\end{array}$ & $\begin{array}{l}\text { Nanocrystalline } \\
\text { materials } \\
\text { Nanotube reinforced } \\
\text { nanocomposites } \\
\text { Nanostructured }\end{array}$ \\
& & & $\begin{array}{l}\text { Naners } \\
\text { multilayer }\end{array}$ \\
\hline
\end{tabular}

Plasma electrolysis can produce any kind of nanostructure. As described, 2-D nanostructures can be divided into two groups, which are also accessible by plasma electrolysis. The most investigated usage of plasma electrolysis is its fabrication ability for 2-D nanostructures, but all of its abilities to fabricate different kinds of nanostructures have the potential to become industrialized. Figure 1.4 illustrates titania nanorods that have been produced by cathodic plasma electrolysis.

\section{3}

\section{Ability of Plasma Electrolysis in Nanostructure Fabrication}

The ability to control the fabrication methods for producing different nanostructures is very important for the development of their future industrial applications. The top-

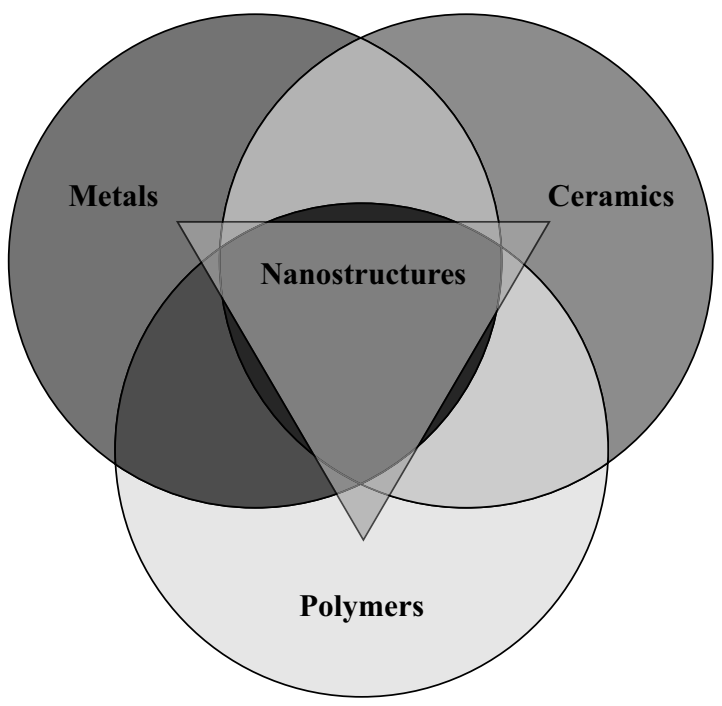

Figure 1.3 Position of nanostructures in engineering materials (areas of covered surfaces are not related to actual contributions). 


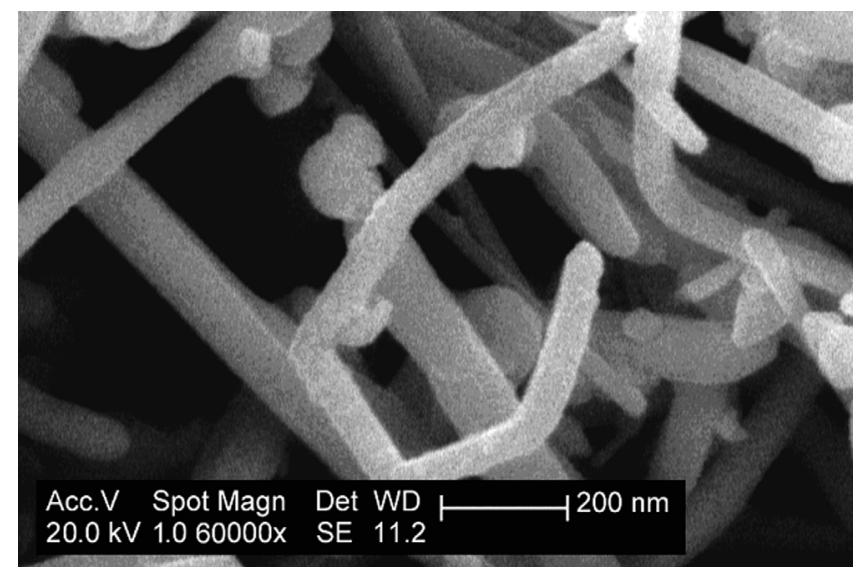

Figure 1.4 Titania nanorods that have been produced by cathodic plasma electrolysis [14].

down and bottom-up approaches for manufacturing nanostructures are well defined in the literature. These classifications and the controllability of different manufacturing methods will help us to better design an industrial process. The most important characteristics of nanostructures are their distribution, average size of nanoparticles (average size of nanocrystallite), size-affected dimension, free active surface, and so on. The accuracy of control and reproducibility of nanostructures with desirable properties are also very important in the evaluation of a method. After these considerations, the ability of the method to be combined with other fabrication methods is also important. Also, from an economic point of view, a method must be comparable with other methods. Exceptions can be considered for methods that have a very high precision for control or a superior feature related to the process.

Precise control of the fabrication method of nanostructures on surfaces is a huge challenge. The final target is atomic fabrication of layers with the precision of one single atom. In this ideal method, atoms will sit beside each other to fabricate a monolayer. Some advances have been achieved by scanning probe microscopy to modify surfaces in this way. Fabrication methods of nanostructures that are near this ideal model are worth exploring on laboratory or industrial scale. After atomic arrangement of the nanostructure, the presence of nanopores and controlling the mentioned different characteristics of nanoparticles (nanocrystallites) as the building blocks of nanostructure is very important. In fact, fabrication methods that also can control these characteristics are of great interest. On the other hand, manufacturing nanostructures with thicknesses larger than a single atomic layer is very important, and various properties (such as electronic, magnetic and electrochemical properties) of such nanostructures depend upon their crystalline order. As mentioned before, in fabricating 2-D nanostructures on surfaces, the desirable method is layer-by-layer growth for the possible formation of single crystals in the nanometric size range.

Plasma electrolysis is a new branch of plasma-assisted surface engineering processes in which small atoms can be embedded into different metal lattices and 
can also fabricate a nanocrystalline layer over its surface. These nanostructured coatings will improve the hardness, wear resistance and corrosion resistance of substrates. Various tiny glow discharges that are produced rapidly on the surface of an immersed component and chemical reaction at elevated temperatures can produce nanostructures with desirable characteristics. Faster diffusion of small atoms toward the surface of a metallic substrate allows the coating process to proceed at lower temperatures and for shorter treatment times. Comparing this method with other methods of diffusion coatings, such as plasma nitriding, plasma immersion implantation, radiofrequency plasma diffusion, low-pressure plasma-assisted treatment, ion beam diffusion, and active screen plasma diffusion, this method can produce hard layers with nanocrystalline structures. As described earlier, these nanostructures show nanometric size-related properties, with a strong relation among the characteristics of their nanostructures and their achieved properties. Also these characteristics can be controlled by precise adjustment of the effective factors of the coating process, such as the duty cycle and the frequency of applied pulsed current.

Beside 2-D nanostructures fabricated by plasma electrolysis, which have been investigated more than other kinds of nanostructures, plasma electrolysis can produce all other kinds of nanostructures. This treatment has been used for the fabrication of titanium carbide nanopowders, titania nanorods, bulk nanostructures such as titanium carbide/tungsten carbide (TiC/WC) nanocomposites, and thick nanocrystalline titanium carbonitride coating. Rapid quenching of the fabricated phase at elevated temperature by tiny sparks in the electrolyte will cause this method to maintain an average size of nanocrystallites of even less than $10 \mathrm{~nm}$. Accelerating high-energy atoms from the electrolyte toward the surface of the substrate will cause this method to act very much faster than similar methods with higher underlayer penetration depth.

One of the most important features of this method for the fabrication of nanostructures is the strong relation among its effective factors and the nanometric size-related properties. Comparing this method with other methods for the fabrication of nanostructures, this method shows a strong ability for industrial lines. Controlling the effective factors of this method is easy, while its setup is relatively cheap, and it can treat the complex shapes of industrial components. This method, from some points of view, is very similar to regular electroplating for fabrication of 2-D and 3-D nanostructures. As the application of electroplating has been done by many industrial units, this method can also use them for its production line, with some changes. The capability of producing nanostructures by this method is more than just electrodeposition, as this method can control the achieved characteristics of the nanostructures more than in electroplating, and also it can produce hard ceramicbased layers faster than by electroplating. As the ability of this method to produce nanostructures is completely based on precisely adjusting its effective factors, and it has relatively numerous effective factors, their optimization for achieving the desired nanostructure is very important. Plasma electrolysis shows great potential for controlling the characteristics of nanostructures with the usual economic considerations and relatively good ability for combination with other methods. Duplex 


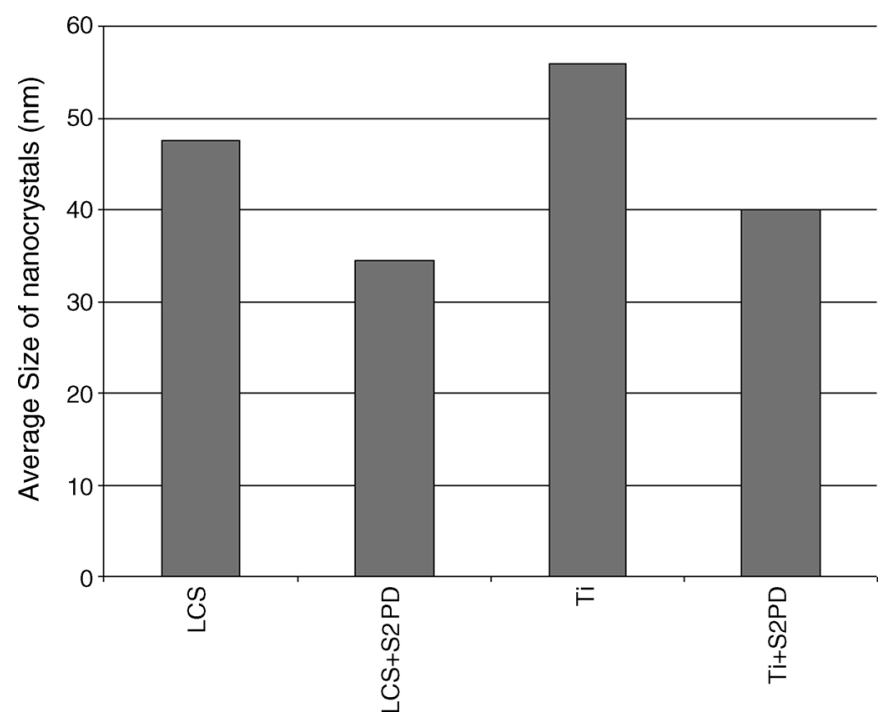

Figure 1.5 Average size of nanocrystalline carbides of different substrates with and without NSSPD process (S2PD in the figure) [15].

methods for the fabrication of nanostructures with considerable effect on nanometric size-related properties can be applied with plasma electrolysis. In fact, the ability of plasma electrolysis to be used in combination with other methods can make it more suitable for its performance in industrial lines. Figure 1.5 illustrates the changes in the average size of carbide-based nanocrystallites fabricated by plasma electrolysis on different substrates $(\mathrm{Ti}=$ titanium and $\mathrm{LCS}=$ low-carbon steel) and also duplex treatments of plasma electrolysis and nanocrystalline surface severe plastic deformation (NSSPD) process.

\section{4}

\section{Relation Between Plasma Electrolysis and Nanotechnology}

Considering 2-D nanostructures, plasma electrolysis can be used for the fabrication of nanostructured coatings on the surface of different kinds of metals/alloys and is usually used for the fabrication of complex layers such as graphite and oxides. Figure 1.6 illustrates the schematic diffusion of elements into a metallic lattice and the fabrication of a nanostructured compound layer and stressed underlayer by cathodic plasma electrolysis. It has been proved that the sizes of most structures produced by this method are in the range of $1-100 \mathrm{~nm}$, which can be called nanostructures, but an important issue that connects these kinds of nanostructures to nanotechnology is that, by changing various characteristics of these nanostructures (e.g. average size of nanocrystallites), the obtained properties of these layers vary greatly. It has been observed for most of these layers that, by lowering the average 


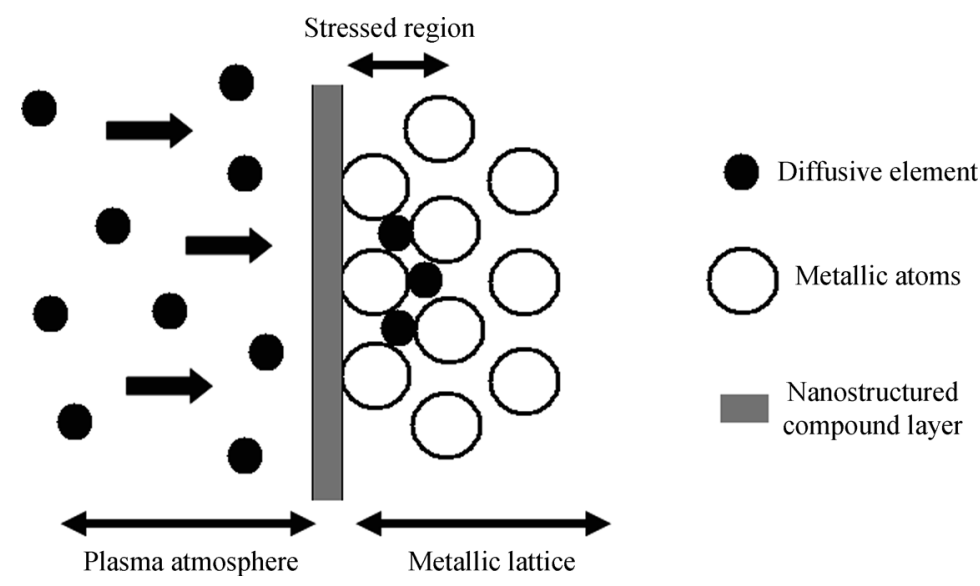

Figure 1.6 Schematic diffusion of elements into a metallic lattice and the fabrication of a nanostructured compound layer and stressed underlayer.

size of nanostructures, their different properties (such as corrosion resistance and wear resistance) will improve significantly. This method can also be applied for surface cleaning of different metallic parts. This method seems to be a very promising method for the fabrication of desired nanostructured layers with significant properties. Table 1.2 illustrates the conditions of the sample treated with plasma electrolysis, the results of which are shown in Figure 1.7. This figure shows

Table 1.2 Central composite design plan in coded values and observed response.

\begin{tabular}{lcccc}
\hline $\begin{array}{l}\text { Experiment } \\
\text { run no. }\end{array}$ & $\begin{array}{c}\text { Ratio of duty cycle } \\
\text { of cathodic direction } \\
\text { to duty cycle of } \\
\text { anodic direction }\end{array}$ & $\begin{array}{c}\text { Frequency of } \\
\text { pulsed current }\end{array}$ & $\begin{array}{c}\text { Treatment } \\
\text { time }\end{array}$ & $\begin{array}{c}\text { Average sizes } \\
\text { of nanocrystallites } \\
\text { (nm) }\end{array}$ \\
\hline 1 & -1 & -1 & -1 & 55.2 \\
2 & 1 & -1 & -1 & 85.4 \\
3 & -1 & 1 & -1 & 32.5 \\
4 & 1 & 1 & -1 & 62.6 \\
5 & -1 & -1 & 1 & 66.1 \\
6 & 1 & -1 & 1 & 95.1 \\
7 & -1 & 1 & 1 & 42.4 \\
8 & 1 & 1 & 1 & 72.6 \\
9 & 0 & 0 & 0 & 58.2 \\
10 & 0 & 0 & 0 & 58.5 \\
11 & -1 & 0 & 0 & 42.9 \\
12 & 1 & 0 & 0 & 72.6 \\
13 & 0 & 0 & -1 & 52.6 \\
14 & 0 & 0 & 1 & 63.4 \\
15 & 0 & -1 & 0 & 75.9 \\
16 & 0 & 1 & 0 & 51.7 \\
\hline
\end{tabular}



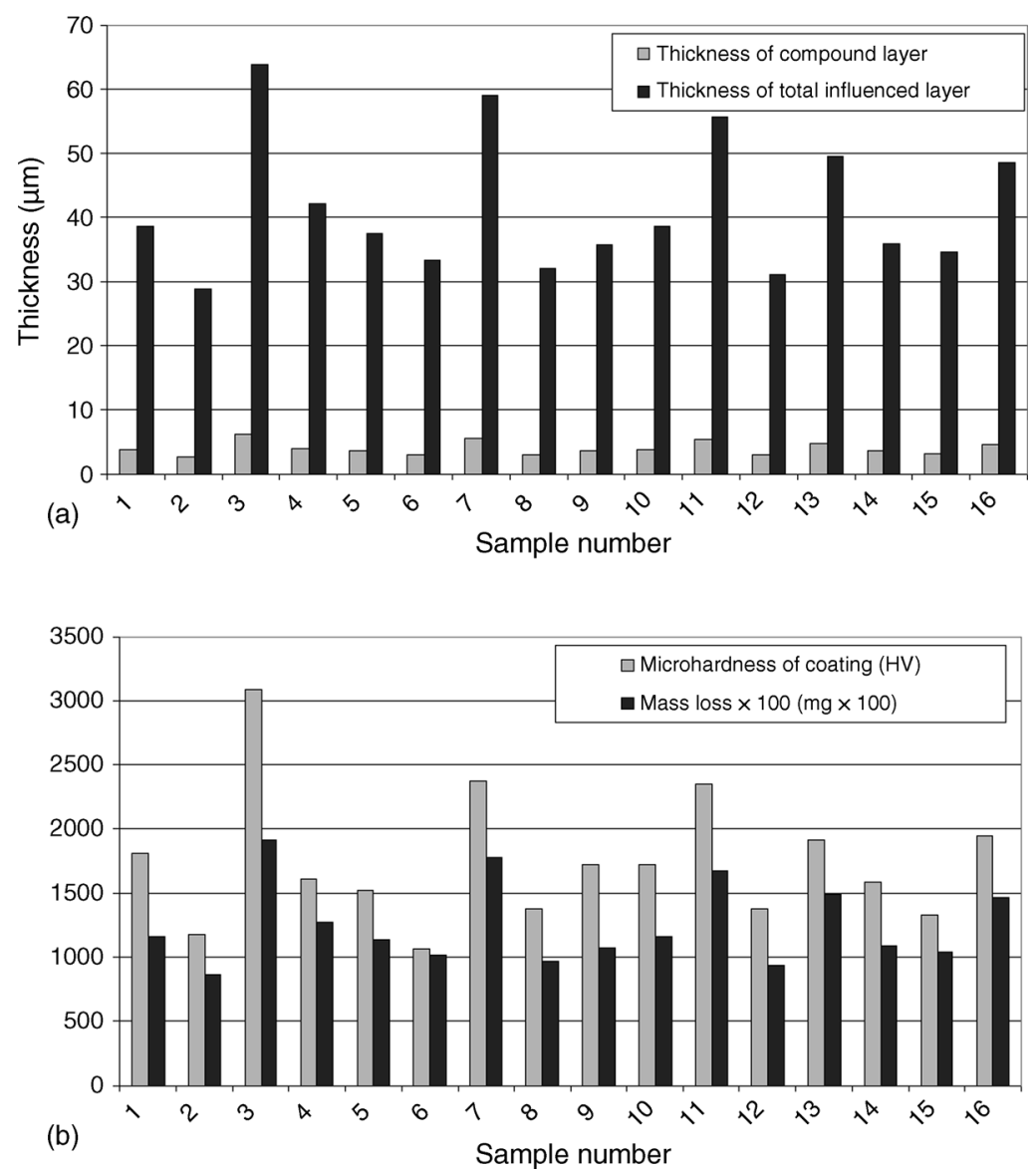

Figure 1.7 (a) Thicknesses of compound layer and total influenced layer of different treated samples. (b) Microhardness and mass loss $(\times 100)$ of different treated samples [16].

the uniform change of enough tested samples to confirm the strong relation between average nanocrystallite size and achieved properties. The coded values in the table $\left(-1,0\right.$ and 1 , respectively) for $\mathrm{X}_{1}, \mathrm{X}_{2}$ and $\mathrm{X}_{3}$ (ratio of duty cycle of cathodic direction to duty cycle of anodic direction $\left(\mathrm{X}_{1}\right)$, frequency of pulsed current $\left(\mathrm{X}_{2}\right)$ and treatment time $\left.\left(\mathrm{X}_{3}\right)\right)$ are $(0.2,0.3,0.4 ; 5,10,15$; and $10,20,30)$.

The anodic branch of plasma electrolysis - usually known as plasma electrolytic oxidation (PEO) or micro-arc oxidation (MAO) - can be used for preparing an oxide layer on the surface of light metals/alloys such as aluminum, titanium, and magnesium. These kinds of coatings are usually used to enhance some of the properties of light metals/alloys, such as wear resistance, corrosion resistance, thermal barrier, and so on. According to the applied conditions of the coating process, the size of the achieved coating structure for these layers varies in the range from a few nanometers up to several micrometers. There are also some reports on the fabrication of nanocomposite layers that can be produced by this method. 


\section{5}

\section{Growth Process of Nanostructured Films}

In recent years, the growth processes of nanostructures have been studied by many researchers due to their strong nanometric size-related physical, mechanical, chemical, and electrochemical properties. The ability of an atom or nanometric particle to move into the surface of a substrate, to arrange itself, and then to fabricate a specific kind of nanostructure mainly depends on its kinetic energy. Deposition methods characterized by high-energy atoms or nanometric particles generally will fabricate dense and flat surfaces, while fabrication of 2-D nanostructures with low-energy atoms or nanometric particles work by agglomeration of nanoclusters on the surface of the target.

On the other hand, plasma electrolysis is an atmospheric pressure-based plasma deposition technique for the fabrication of nanostructures. The plasma-gas interactions are very complex, and the loss and dispersion of the kinetic energies of the atoms or nanometric particles involved in the plasma are just one of the factors. As a result, 2-D nanostructure growth is concerned in a complex manner with gas properties such as pressure.

Carbon-based nanostructures show interesting size-dependent mechanical and physical properties. Two-dimensional carbon nanostructures can be produced by cathodic arc processes or direct current (DC) magnetron sputtering with high flow of related gas. Embedding of 3-D nanostructures mainly consists of different planes with fullerene-like properties, which will cause fabrication of nanostructures with superior mechanical properties. Their nanostructure will form by a dense and compressed array of rounded graphene planes like turbostratic carbon but with a nanometric twist.

Considering plasma electrolysis as an electrolyte-based method, the fabrication methods of nanostructures will divide here into two groups (electrolyte-based methods and non-electrolyte-based methods), with some branches in each group. These methods will be studied by considering methods similar to plasma electrolysis and their differences will be discussed.

\section{6}

\section{Electrolyte-Based Methods}

Different liquids, especially water, are good solvents, with high permittivity for polar or ionic materials. Therefore, a lot of chemical reactions will occur in the aqueous phase. Various kinds of nanopowders for different applications can be fabricated by adding liquid agents to aqueous electrolytes of related salts with specific $\mathrm{pH}$. Amorphous nanostructures or nanocrystalline structures and nanocomposites can also be fabricated using aqueous solutions. For example, reduction of aqueous potassium borohydride was used for the fabrication of amorphous Fe-Co-B ultrafine powders for use in ferrofluid systems and magnetic memory. The amorphous phase was created when the reaction was carried out below the glass transition 
temperature and became constant with a high concentration of atomic boron. Reaction media often oblige us in choosing the kind of fabricated product(s). In using sodium borohydride for the preparation of $\mathrm{Co}-\mathrm{B}$ alloy, the solvent plays an important role in determining the final product. In aqueous media, $\mathrm{Co}_{2} \mathrm{~B}$ nanoparticles were the initial product; and in non-aqueous reduction of cobalt ions in diglyme, cobalt nanoparticles were formed. Reductions of aqueous and non-aqueous borohydride were also considered for the fabrication of $\mathrm{Fe}, \mathrm{FeB}$, and $\mathrm{Fe}_{2} \mathrm{~B}$ nanoparticles. Metastable phases can be created in a chemical reaction by fast kinetics. For example, $\mathrm{Fe}$ and $\mathrm{Cu}$ are insoluble in the stable state. Metastable alloys of iron and copper can be fabricated by using far-from-stable processing techniques, such as rapid cooling of the vapor phase or mechanical alloying. Nanocrystalline $\mathrm{Fe}_{x} \mathrm{Cu}_{100-x}$ ( $x$ is atomic percent, at\%) alloys and nanocomposite powders were fabricated by aqueous solution reduction of iron chloride and copper chloride (in different molar ratios) by sodium borohydride solution. This reaction was done at room temperature with continuous stirring for five minutes. The ratio of iron and copper atoms is near to that in the original aqueous electrolytes. When $x=40$, only face-centered cubic (fcc) metastable alloys were fabricated. At higher iron concentration such as $x=70$, phase separation of fcc copper and body-centered cubic (bcc) iron will happen. The formation of copper oxide was observed and its concentration is related to that of copper. Nanopowders were agglomerations of nanocrystallites. Nanocrystallites were between 30 and $45 \mathrm{~nm}$ for alloys and 10-15 $\mathrm{nm}$ for iron and 30-40 $\mathrm{nm}$ for copper in the nanocomposites. The fabrication of the nanocrystalline phases was controlled by decreasing the boron concentration in the nanopowders when a higher molarity of borohydride was considered in the specified reaction.

Another example is the system of cobalt and copper, which creates solid solutions. Nanopowders of $\mathrm{Co}_{x} \mathrm{Cu}_{100-x}$ were synthesized by sodium borohydride reduction of aqueous cobalt and copper chloride solutions. The as-synthesized nanopowders were a combination of fcc and amorphous phases. The concentration of the amorphous phase increased with the ratio of cobalt to copper. The annealed nanopowders phase separated into fcc cobalt and fcc copper at about $500^{\circ} \mathrm{C}$. Annealing caused considerable surface sintering and some grain growth $(30-40 \mathrm{~nm})$, and boron impurity was seen to separate at the surfaces of the sintered nanopowders.

Although electrolyte-based methods for fabricating nanostructures are not new, their use in the synthesis of different nanostructures requires special attention to prevent undesirable products. Impurities, salts, and other reaction products may not be totally removed even with washing, if they are embedded into the nanoparticles or agglomerations during a fast chemical reaction. Washing to remove soluble salts may lead to hydrolysis and oxidation of nanoparticles. Post-drying often needs vacuumbased treatments to prevent oxidation.

\subsection{1}

\section{Electrodeposition}

Deposition of a pure metal or co-deposition of an alloy in electrodeposition from an electrolyte takes place on the surface of the target, which is the cathode of the 
electrochemical system of the coating process. This process will happen by applying an external (direct or pulsed) current to the electrodeposition system. The substrate must be electrically conductive. By this method, 2-D and 3-D nanostructures of metals, alloys, and nanocomposites have been deposited successfully. It can produce very dense and approximately porosity-free nanostructures without any requirement for a subsequent densification process. Nanostructured grains were plated when electrodeposition effective factors, such as electrolyte composition, $\mathrm{pH}$, duty cycle, frequency, temperature and average current density, were controlled. Adjusting these factors will cause nucleation of grains over previously formed grains. Plasma electrolysis is a completely electro-based method, and its cathodic branch is very similar to this process, with some more possibilities for fabricating new nanostructures $[17,18]$.

\subsection{2}

\section{Electroless Deposition}

In contrast to the case of electrodeposition, in this method, electrons are produced by chemical reactions with no supply of exterior current. Unlike electroplating, substrates can be electrical insulators. Electroless deposition can take place by the following mechanisms:

1. deposition by ion or charge replacement

2. deposition by contacting the coated metal

3. autocatalytic deposition.

In the third mechanism, a non-catalytic surface for electroless deposition is first coated with catalyst particles. Catalyst particles are typically colloids such as palladium (with diameter of at least $2 \mathrm{~nm}$ ) encapsulated in a tin oxide case.

Electroless deposition can take place in aqueous and non-aqueous electrolytes. These methods were used for the fabrication of different nanostructures on the surfaces of metallic and non-metallic substrates. Hydrolysis of the surface in aqueous solutions will produce some problems for the deposition process. The nonaqueous process can produce nanostructure deposits and can be used with watersensitive substrates or supporting phases in nanocomposites. For example, copper coatings were fabricated on aluminum nitride substrates, which will react with water [19-21].

\subsection{3}

\section{Plasma Electrolysis}

Plasma electrolysis is a relatively new method that has been shown to be appropriate for enhanced properties for different metallic substrates, especially for their double protection of corrosion and thermal barrier applications. This electrolyte-based method is mostly called micro-arc oxidation (MAO) [22] or plasma electrolytic oxidation (PEO) [23] in its anodic branch, and plasma electrolytic saturation 
(in general) for its cathodic branch [24]. Coatings fabricated on some aluminumbased alloys making use of this method exhibit superior anti-corrosion and mechanical properties, which have meant that this method has developed successfully for industrial applications.

This method also has the advantage of, and ability for, easy and rapid fabrication of novel (micro- and nano-) composite coatings by the addition of an appropriate nanopowder of a second (supporting) phase in the electrolyte and making a suspension. These nanocomposites can increase the mechanical properties of metallic substrates. In fact, nanocomposite coatings produced by the addition of appropriate nanopowders not only increased the mechanical properties of the layers but also enhanced their other properties, such as corrosion resistance and thermal conductivity.

\section{7}

\section{Non-Electrolyte-Based Methods}

\subsection{1}

\section{Hydrolysis}

Sometimes precipitation from an electrolyte will cause fabrication of an insoluble hydroxide that can be changed to its oxide by heat-aided dehydration. Chemical methods for fabrication of monodispersed colloidal microparticles have been investigated in different papers. For example, metal (hydrous) oxide particles were fabricated by hydrolysis involving adjusted deprotonation of hydrated cations. Covered colloidal particles can also be fabricated by using hydrolysis. Controlling the hydrolysis of aluminum salts, where solute complex agents were shaped instead of precipitation, materials such as hematite, chromia and titania were covered with a layer of aluminum (hydrous) oxide. Deposition of the coating occurred by surface precipitation, which was adjusted by the available surface area of the particle substrate and the concentrations of the complex agents creating the coating. The thickness of the coating was changed by the mass parameters and treatment time of the process $[25,26]$.

\subsection{2}

\section{Hydrothermal}

In hydrothermal methods the reaction mixture is heated at temperatures above the boiling point of water in a closed system such as an autoclave and the sample is treated by steam at high pressure. Precipitation of zirconia under hydrothermal conditions will lead to nanopowders with a narrow size distribution and adjustable composition and nanostructure. Treating $\mathrm{Zr}\left(\mathrm{SO}_{4}\right)_{2}$ by this method at $250^{\circ} \mathrm{C}$ in the presence of $\mathrm{MgSO}_{4}$ or $\left(\mathrm{NH}_{4}\right)_{2} \mathrm{SO}_{4}$ caused the fabrication of acicular monoclinic zirconia. Zirconia fine particles have dimensions around $0.3-1.3 \mu \mathrm{m}$ in length and $0.1-0.2 \mu \mathrm{m}$ in width $[27,28]$. 


\subsection{3}

\section{Sol-Gel Methods}

The sol-gel method is promising for the fabrication of different kinds of nanostructures. A lot of research has been done on sol-gel-derived nanostructures. Some related ones will be discussed here. The history of this method goes back more than a century.

Nanostructured $\mathrm{TiO}_{2}$ thin films are used in a wide range of applications. These applications consist of ultraviolet filters for optics and packing materials, antireflection coatings for photovoltaic cells and passive solar collectors, photocatalysts for the purification and treatment of water and air, anodes for ion batteries, electrochromic displays, transparent conductors, self-cleaning coatings of windows and tiles, humidity sensors, gas sensors, and barrier layers for corrosion protection. Several techniques have been used for the preparation of transparent $\mathrm{TiO}_{2}$ thin films, which include sputtering chemical vapor deposition, pulsed laser deposition, laser molecular-beam epitaxy method, and sol-gel technique. The sol-gel technique has distinct advantages over the other techniques due to excellent compositional control, homogeneity on the molecular level due to the mixing of liquid precursors, and lower crystallization temperature. Moreover, the microstructure of the film (i.e. pore size, pore volume, and surface area) deposited by the sol-gel process can be tailored by the control of process variables, and a more generic approach to enhance corrosion resistance is to apply protective films or coatings without cracks. The presence of cracks and defects in a coating, with increasing current density, will lead to localized corrosion.

Recently, a sol-gel-based titania coating has been developed and characterized on stainless steel and mild steel substrates to improve their abrasion resistance and corrosion resistance properties. The precursors were selected due to their specific physico-chemical properties. The alkoxide precursor was selected because precursors containing long-chain hydrocarbon groups are hydrophobic, enable better mixing of the organic functionalities at the molecular level, and promote the formation of non-porous gels, so that the final film will have good properties. When appropriate chemical composition and processing conditions are applied, relatively dense organic-inorganic hybrid coatings can be achieved for applications including sensors, anti-reflection coatings, wear resistance and corrosion protection.

There are many ways to reduce the percentage of cracks in the final coat produced with the sol-gel method, some of which include the use of:

1. polymeric agents, such as polyethylene glycol, polyvinyl alcohol, polyvinyl propylene

2. acetic acid

3. ethyl acetoacetate and controlling the heat treatment cycle

4. the hybridization process.

In the first and second methods, the rate of vaporization of volatile organic agents from the coating will decrease significantly. Also, increasing porosity and specific 
surface area of the coating will lead to a significant decrease in the amount of cracks in the coating.

In the third way, chemical agents act like a binder and adsorb titanium compounds around themselves. Whenever the sample is rinsed in solution, these compounds will bind toward the sample and hence the percentage of coated area will increase. After evaporation of the organic agents, the crack percentage will decrease. The evaporation rate and obtained stress in the coating will be controlled by controlling the heat treatment conditions [29].

In the fourth method, the coating produced will be exposed to a heat treatment at $550{ }^{\circ} \mathrm{C}$ for $10-30 \mathrm{~min}$, then to distilled water at a temperature of $100^{\circ} \mathrm{C}$, and finally to a low-temperature heat treatment cycle. In this method, the hybrid compounds formed will have a significant decrease in cracks. Figure 1.8 illustrates an atomic force microscopy (AFM) surface morphology nanograph of $\mathrm{TiO}_{2}$ nanoparticle coating on mild steel produced by the sol-gel method. The $\mathrm{TiO}_{2}$ nanoparticle sizes were about 40-60 nm, as obtained from X-ray diffraction (XRD) and AFM.

Achieving a high-quality coating is related to different parameters of solution and heat treatment. The temperature of $550^{\circ} \mathrm{C}$ has been applied to obtain the best coating quality possible at high temperatures. Anatase was determined by heat treatment at $550{ }^{\circ} \mathrm{C}$ on the surface of the sample. $\mathrm{ATiO}_{2}$ nanoparticle coating with a thickness of $561 \mathrm{~nm}$ will shift the open-circuit potential about $320 \mathrm{mV}$. Increasing the coating thickness up to $561 \mathrm{~nm}$ will improve the corrosion properties of the
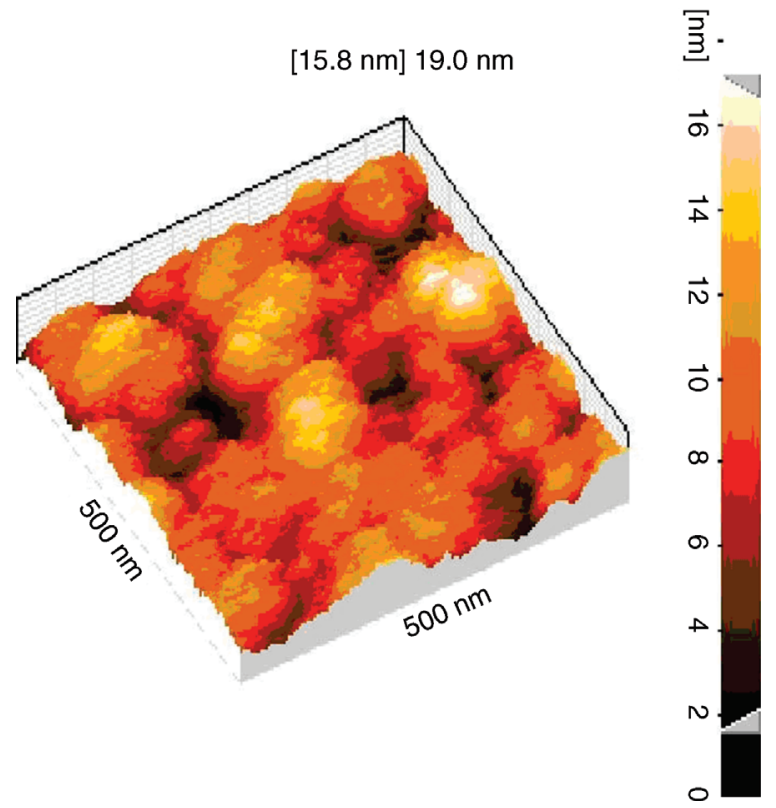

Figure 1.8 AFM surface morphology nanograph of $\mathrm{TiO}_{2}$ nanoparticle coating on mild steel by the sol-gel method [29]. 


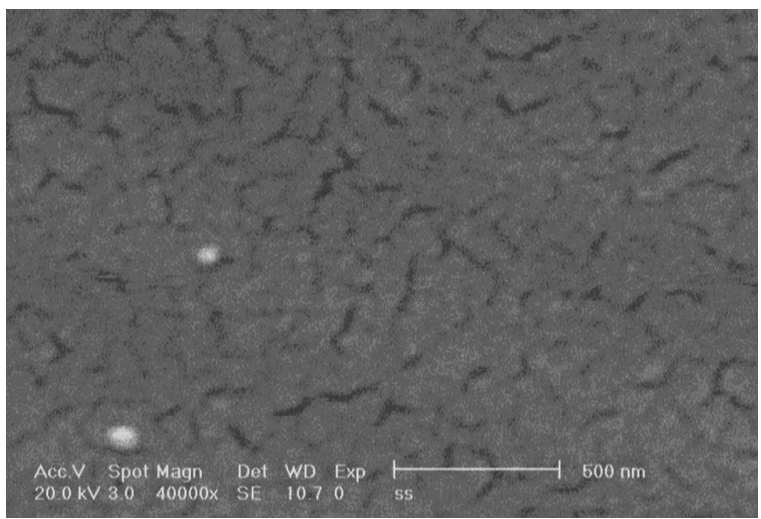

Figure 1.9 SEM micrograph from the surface of a coating obtained with the following effective factors: $9 \mathrm{~mm} / \mathrm{s}$ dip coating rate, $3 \mathrm{~h}$ drying time, $1^{\circ} \mathrm{C} / \mathrm{min}$ heat treatment rate and three dip coatings [30].

coated sample. The corrosion resistance of mild steel by performing this coating will improve about 190 times over that of the raw substrate. Increasing the coating thickness to more than $561 \mathrm{~nm}$ will decrease the physical and corrosion properties of the coated sample compared to those of samples with a coating thickness less than $561 \mathrm{~nm}$. The $\mathrm{TiO}_{2}$ nanoparticle coating with capacitive properties will decrease the corrosion current density of mild steel. Figure 1.9 illustrates the scanning electron microscopy (SEM) nanostructure of the surface of a coating obtained by choosing the following effective factors: $9 \mathrm{~mm} / \mathrm{s}$ for dip coating rate, $3 \mathrm{~h}$ for drying time, $1^{\circ} \mathrm{C} / \mathrm{min}$ for heat treatment rate, and three times of dip coating [30].

Another successful example of using the sol-gel method for fabrication of nanostructures concerns cerium oxide nanostructures. The natural oxide film on aluminum does not offer sufficient protection against aggressive anions, so inhibitors and coatings are used to improve the protective features of its surface. Among these replacements, rare-earth compounds, particularly cerium compounds, have attracted significant attention. Several researchers have demonstrated that treatments with aqueous solutions of cerium salts effectively inhibit the corrosion of aluminum alloys. Conversion coatings were obtained by prolonged immersion in hot aqueous solutions of rare-earth ions. Recently, an economically comparable conversion coating process based on the use of water-based solutions of cerium chloride and hydrogen peroxide was developed to produce conversion coatings on aluminum alloy in shorter times.

Use of sol-gel methods for metallic surface treatments is a relatively new approach in surface engineering. The sol-gel method consists of simultaneous hydrolysis and condensation reactions. Prior to gelation, the sol is ideal for preparing thin films by common processes, including dipping, spinning or spraying. These application methods generally produce thin films of sub-micrometer thicknesses. Preparation of thicker films is possible through the application of multiple layers or by variation in withdrawing or spinning speed in the case of dip and spin coating, respectively. 

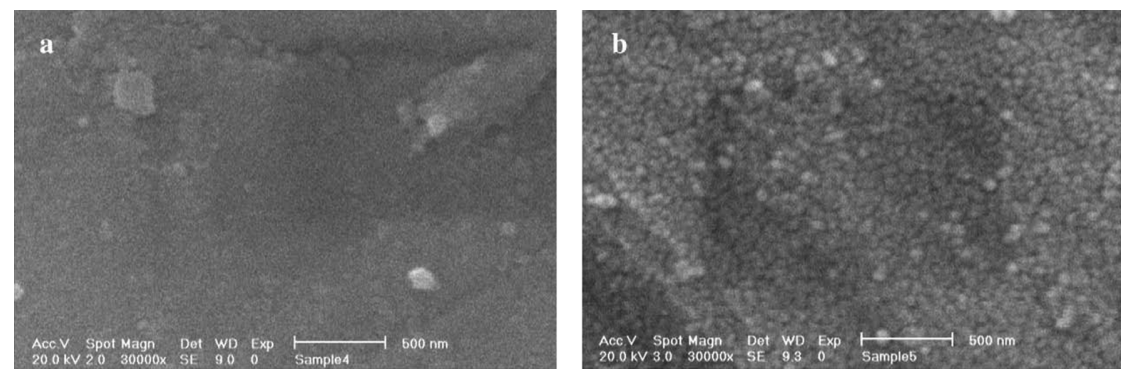

Figure 1.10 Topographies of the nano-sized cerium oxide films that were heat treated at (a) $300^{\circ} \mathrm{C}$ and (b) $400^{\circ} \mathrm{C}$ [31].

Cerium oxide was prepared by dip immersion and sol-gel methods on AA5083H321 aluminum alloy. The surface topographies of films that were heat treated at different temperatures are shown in Figure 1.10. Highly uniform and crack-free cerium oxide nanofilms were deposited at different temperatures. The film that was deposited at $300^{\circ} \mathrm{C}$ is characterized by non-crystallized topography, whereas the film deposited at $400^{\circ} \mathrm{C}$ is characterized by a nanocrystallized structure. This shows that nano-sized cerium oxide crystallized between 300 and $400^{\circ} \mathrm{C}$. The thickness of the conversion nano-sized cerium oxide films (by sol-gel method) without pretreatment is about $150-200 \mathrm{~nm}$. The average grain size of the cerium oxide coating (obtained by sol-gel method) is about $15 \mathrm{~nm}$. The crystallite sizes of films obtained with different acetic acid ratios on the surface of AA7020-T6 aluminum alloy were determined by using the Scherrer equation (Figure 1.11) and it was seen that, by increasing the acetic acid ratio, the mean average size of the nanocrystalline grains decreased [31, 32].

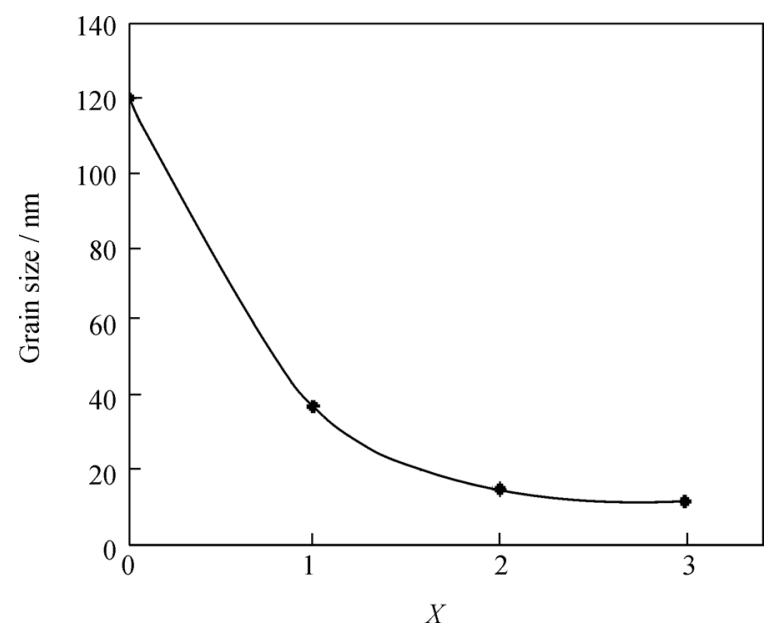

Figure 1.11 Curve of grain size of the cerium oxide nanofilm with different acetic acid $/ \mathrm{CeCl}_{3}$ molar ratios $(X=0,1,2,3)$ fabricated by the sol-gel method [32]. 
$20 \mid 1$ Synthesis and Processing of Nanostructured Films

1.8

Introduction to Plasma Electrolysis

The formation of nanostructured and nanocomposite layers by water-based methods containing different nanoparticles and ions from coating baths has also been reported in different papers. Research on these kinds of nanostructured and nanocomposite layers has increased during recent years due to the fact that nanostructured and nanocomposite layers can give desirable properties to the coated component, such as high wear resistance, high corrosion resistance, good oxidation resistance, and self-lubrication. Electrolytic fabrication of nanostructured and nanocomposite layers, then, is focused towards the optimization of their effective factors, that is, current density, electrolyte temperature used, nanoparticle concentration and bath composition.

Various metal- and ceramic-based nanostructured and nanocomposite layers have been successfully produced by electrolyte-based methods. Nanostructured and nanocomposite layers have been obtained by aqueous electroplating of different nanoparticles, such as $\mathrm{Al}_{2} \mathrm{O}_{3}, \mathrm{SiC}$, and $\mathrm{TiO}_{2}$. Numerous reports have been devoted to electroplated nanocomposites, and they have studied the influence of the alloying matrix on the electroplating process and the properties of the obtained nanocomposite layers.

Different plasma treatments are increasingly used for the surface modification of metallic components, as these methods are environmentally friendly. One that has recently attracted the attention of researchers is plasma electrolysis, which can be classified under atmospheric pressure plasma treatments. Like most other electrochemical coating methods, this process basically consists of sample immersion in an electrolyte with a relatively high applied potential (usually 200-2000 V). During this process, the surface of the sample is subjected to continuous sparking and, based on the used electrolyte and applied mode of current, different nanostructured coatings such as oxides, carbides, carbonitrides, and so on can be produced.

\section{References}

1 Lee, Y.S. (2008) Nanostructured materials, in Self-Assembly and Nanotechnology: A Force Balance Approach, John Wiley \& Sons, Inc., Hoboken, NJ, pp. 183-219.

2 Stampfer, C. and Hierold, C. (2008) Electromechanical carbon nanotube transducers, in Carbon Nanotube Devices: Properties, Modeling, Integration and Applications (ed. C. Hierold), Wiley-VCH, Weinheim, pp. 43-81.

3 Kreupl, F. (2008) Carbon nanotubes in microelectronic applications, in Carbon Nanotube Devices: Properties, Modeling, Integration and Applications (ed. C. Hierold), Wiley-VCH, Weinheim, pp. 1-41.

4 Eddaoudi, M. and Eubank, J.F. (2008) Periodic nanostructures based on metal-organic frameworks (MOFs): en route to zeolite-like metal-organic frameworks (ZMOFs), in Organic Nanostructures (eds J.L. Atwood and J.W. Steed), Wiley-VCH, Weinheim, pp. 251-274.

5 Nakahara, T. and Imai, T. (2005) Frontier nanotechnology for the next generation, in Nanotechnology: Global Strategies, Industry Trends and Applications 
(ed. J. Schulte), John Wiley \& Sons, Ltd, Chichester, pp. 128-135.

6 Homma, T. (2007) Electrochemical fabrication of arrayed nanostructures, in Electrocrystallization in Nanotechnology (ed. G. Staikov), Wiley-VCH, Weinheim, pp. 208-216.

7 Tsuji, N. (2009) Fabrication of bulk nanostructured materials by accumulative roll bonding (ARB), in Bulk

Nanostructured Materials (eds M.). Zehetbauer and Y.T. Zhu), Wiley-VCH, Weinheim, pp. 235-253.

8 Aliofkhazraei, M., Morillo, C., Miresmaeili, R., and Sabour Rouhaghdam, A. (2008) Surface and Coatings Technology, 202, 5493-5496.

9 Aliofkhazraei, M. and Sabour Roohaghdam, A. (2007) Electrochemistry Communications, 9, 2686-2691.

10 Yerokhin, A.L., Nie, X., Leyland, A., Matthews, A., and Dowey, S.J. (1999) Surface and Coatings Technology, 122, 73-93.

11 Aliofkhazraei, M., Sabour Rouhaghdam, A., and Heydarzadeh, A. (2009) Materials Characterization, 60, 83-89.

12 Müller, U. (2007) Nanostructures, in Inorganic Structural Chemistry, 2nd edn, John Wiley \& Sons, Ltd, Chichester, pp. 241-245.

13 Köhler, M. and Fritzsche, W. (2008) Characterization of nanostructures, in Nanotechnology, 2nd edn, Wiley-VCH, Weinheim, pp. 211-224.

14 Mahmood, A., Alireza Sabour, R., Ehsan, G., and Ehsan, M. (2009) Plasma Processes and Polymers, 6, S214-S217.

15 Mahmood, A. and Alireza Sabour, R. (2008) Formation of nanocrystalline layers by surface severe plastic deformation and pulsed plasma electrolytic carburizing, in Thin Films 2008: The 4th International Conference on Technological Advances of Thin Films and Surface Coatings, Singapore, 13-16 July, 2008.

16 Aliofkhazraei, M., Hassanzadeh-Tabrizi, S.A., Sabour Rouhaghdam, A., and Heydarzadeh, A. (2009) Ceramics International, 35, 2053-2059.

17 Alimadadi, H., Ahmadi, M., Aliofkhazraei, M., and Younesi, S.R. (2009) Materials and Design, 30, 1356-1361.
18 Nakanishi, S. (2008) Self-organized formation of layered nanostructures by oscillatory electrodeposition, in Nanostructured Materials in Electrochemistry (ed. A. Eftekhari), Wiley-VCH, Weinheim, pp. 267-290.

19 Crobu, M., Scorciapino, A., Elsener, B., and Rossi, A. (2008) Electrochimica Acta, 53, 3364-3370.

20 Wooddell, M.G., Pickrell, G., and Scott, B. (2009) Increased functionality of novel nano-porous fiber optic structures through electroless copper deposition and quantum dot solutions, in Advances in Energy Materials (eds F. Dogan and N. Manjooran), Ceramic Transactions, volume 205, John Wiley \& Sons, Inc., Hoboken, NJ, pp. 123-134.

21 Sarret, M., Müller, C., and Amell, A. (2006) Surface and Coatings Technology, 201, 389-395.

22 Kim, M.-S., Ryu, J.-J., and Sung, Y.-M. (2007) Electrochemistry Communications, 9, 1886-1891.

23 Wei, D., Zhou, Y., Jia, D., and Wang, Y. (2008) Applied Surface Science, 254, 1775-1782.

24 Aliofkhazraei, M., Sabour Rouhaghdam, A., Heydarzadeh, A., and Elmkhah, $\mathrm{H}$. (2009) Materials Chemistry and Physics, 113, 607-612.

25 Ozawa, M., Suzuki, S., Loong, C.K., and Thiyagarajan, P. (2008) Synthesis and microstructure of powder and porous $\mathrm{ZrO}_{2}$ from hydrolysis process, in Proceedings of the 21st Annual Conference on Composites, Advanced Ceramics, Materials, and Structures - B (ed. J.P. Singh), Ceramic Engineering and Science Proceedings, volume 18, issue 4, John Wiley \& Sons, Inc., Hoboken, NJ, pp. 105-110.

26 Novak, S. and Kosmač, T. (2000) Large alumina parts from an aqueous suspension: hydrolysis assisted solidification (HAS), in Ceramics - Processing, Reliability, Tribology and Wear (ed. G. Müller), WileyVCH, Weinheim, pp. 150-155.

27 Bozo, C., Gaillard, F., and Guilhaume, N. (2001) Applied Catalysis A: General, 220, 69-77.

28 Kanade, K.G., Baeg, J.O., Apte, S.K., Prakash, T.L., and Kale, B.B., (2008) Materials Research Bulletin, 43, 723-729. 
$22 \mid 1$ Synthesis and Processing of Nanostructured Films

29 Shanaghi, A., Sabour, A., Shahrabi, T., and Aliofkhazraee, M. (2009) Protection of Metals and Physical Chemistry of Surfaces, 45, 305-311.

30 Shanaghi, A., Sabour Rouhaghdam, A., Shahrabi, T., and Aliofkhazraei, M. (2008) Materials Science, 44, 233-247.
31 Hasannejad, H., Aliofkhazraei, M., Shanaghi, A., Shahrabi, T., and Sabour, A.R. (2009) Thin Solid Films, 517, 4792-4799.

32 Hasannejad, H., Shahrabi, T., and Aliofkhazraei, M. (2009) Rare Metals, 28, 98-101. 